\title{
A Dirichlet problem with applications to solar prominences
}

\author{
I. McKaig \\ Department of Mathematics, Tidewater Community College, Virginia Beach, VA, USA \\ e-mail: tcmckai@tc.cc.va.us \\ Received 11 October 2000 / Accepted 5 December 2000

\begin{abstract}
Convective motions in the photosphere and sub-photosphere may be responsible for generating the magnetic fields that support long-lived quiescent solar prominences. The connection is explored here by solving a Dirichlet problem on a semi-infinite strip where the base of the strip is the photosphere, and the strip extends into a current free corona. Even though the convection is simulated only by a one-dimensional potential prescribed at the photosphere it is found that both Kippenhahn-Schlüter and Kuperus-Raadu type fields are possible.
\end{abstract}

Key words. supergranulation - convection - prominences

\section{Introduction}

A quiescent solar prominence is a marbled ribbon of plasma suspended thousands of miles above the solar surface. Prominences can last from days to months arching through the hotter more rarified corona and are connected to the photosphere by feet that drop vertically into the chromosphere above supergranule boundaries (Priest 1982). They are grand and amazing structures as borne out by the pictures in Fig. 1 .

Material has been clocked moving from $5-15 \mathrm{~km} \mathrm{~s}^{-1}$ through the filamentry structures that loop through the plasma (Tandberg-Hanssen 1974). As the sun rotates the prominence drifts towards the closest pole, and is deformed and stretched as it goes by differential rotation. The plasma is thought to be supplied to the sheet by either injection from the photosphere or condensation from the corona (Tandberg-Hanssen 1979). It can depart quietly by draining down to the surface through the feet or suddenly in an explosive event lasting only hours, becoming part of the solar wind in a coronal mass ejection. Despite all of this activity the bulk of the lifetime of a prominence is usually modeled as a thin block of plasma supported against gravity and confined by a static magnetic field (see Fig. 2).

Ever since the primary support mechanism has been understood to be magnetic various models have been proposed and modified. Two of the most successful models have been the Kippenhahn-Schlüter (KS) normal polarity model (Kippenhahn \& Schlüter 1957) and the KuperusRaadu (KR) inverse polarity model (Kuperus \& Raadu 1974) (see Fig. 3). The KS model was derived as a magnetostatic solution to the force balance equation, $-\boldsymbol{\nabla} p+\boldsymbol{j} \times$ $\boldsymbol{B}+\rho \boldsymbol{g}=\mathbf{0}$. It is called a normal polarity model since the field below and through the plasma block runs in the same direction. In the KR model the prominence forms in a current sheet and the field above and below the plasma run in opposite directions. Others have extended these models to include energy balance (Poland \& Anzer 1971), variations of the magnetic field with height (Ballester \& Priest 1987), changing the angle the prominence normal makes with the horizontal magnetic field (Milne et al. 1979), or even created their own models to include three dimensional effects such as twisting of the magnetic field (Priest et al. 1989).

The KS model is somewhat phenomenological in nature since the supporting magnetic field is simply placed in the corona with no generating mechanism. Although understood to be initially formed by currents in the convection zone that escape the photosphere there is no direct connection. This can only lead to nonphysical models. In the more physical KR model the photospheric boundary is mimicked by a current filament in the convection zone mirroring the prominence filament, but a detailed field distribution is still not specified. We would like to build static magnetic configurations directly from photospheric flows. This would provide a more direct connection between magnetic fields generated by currents in and below the photosphere and magnetic topologies capable of supporting plasma in the corona. This approach was inspired by the papers of Parker (1963) \& Weiss (1966) where convective flows generate and expel magnetic fields, and Malherbe \& Priest (1983) where ad hoc complex functions were used to describe magnetic configurations. Parker found exact solutions to the induction equation $\frac{\partial \boldsymbol{B}}{\partial t}=\boldsymbol{\nabla} \times(\boldsymbol{v} \times \boldsymbol{B})+\eta \boldsymbol{\nabla}^{2} \boldsymbol{B}$ for various flows $\boldsymbol{v}$ and included time-dependent and steady state cases. Weiss numerically studied the effect of convective flows on initially uniform magnetic fields at various Reynolds numbers. Both 


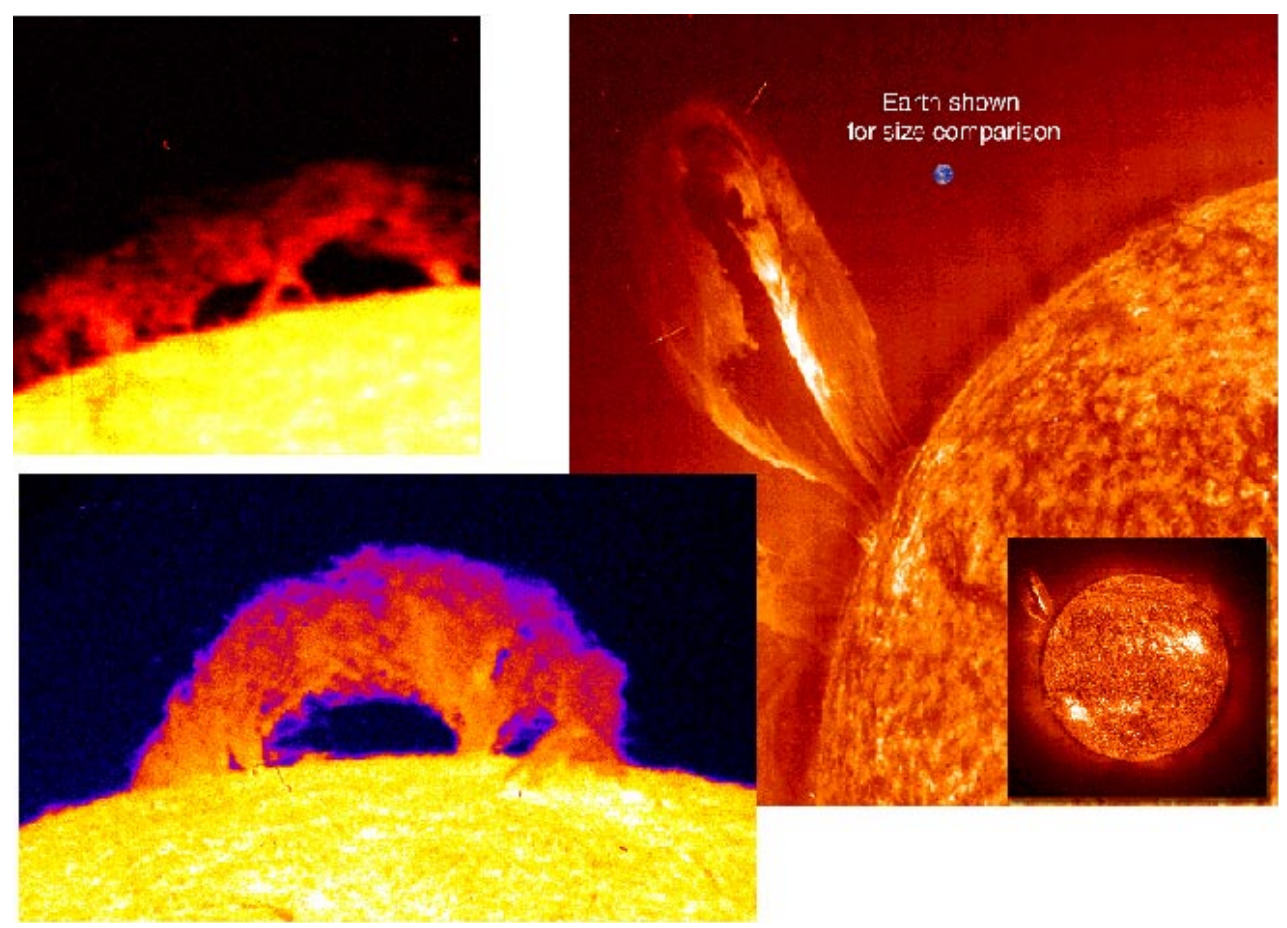

Fig. 1. The left two photographs are courtesy of "Observatoire Midi Pyrénées, INSU/CNRS, France" and show classic quiescent prominences. The right photograph is courtesy of "SOHO/EIT consortium, a project of the ESA and NASA", and shows a prominence in the eruptive phase. For more pictures visit their web sites http://mesola.obspm.fr and http://sohowww.estec.esa.nl

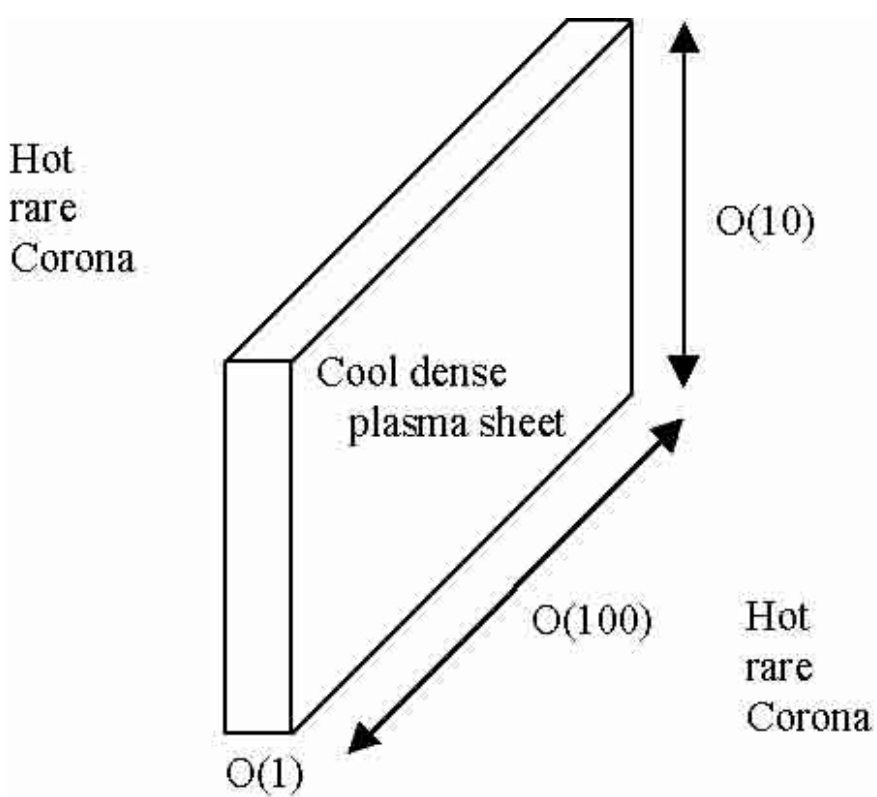

Fig. 2. A solar prominence is often modeled as a block of plasma suspended in a surrounding magnetic field. The typical quiescent prominence has a length of $200000 \mathrm{~km}$, a height of $50000 \mathrm{~km}$ and is $6000 \mathrm{~km}$ wide, but each of these dimensions can vary by a factor of 10 (Priest 1982). The average temperature is about $7000 \mathrm{~K}$, cool compared to the ambient $1000000 \mathrm{~K}$ corona. The density of the sheet is $100-1000$ times greater than that of the corona

authors found that the magnetic field was ejected from regions of high velocity and that even the time dependent
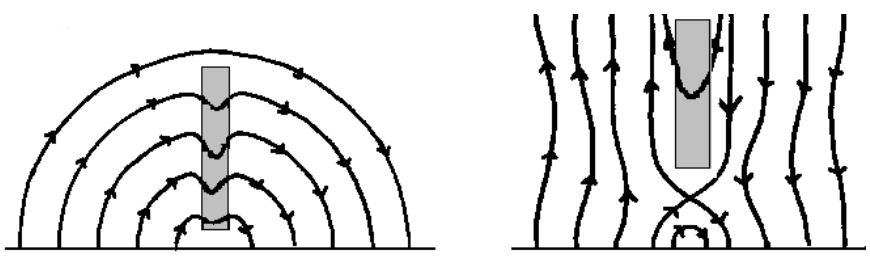

Fig. 3. The above figures show representative KS (left) and KR (right) magnetic configurations. In both models the prominence is between two regions of opposite magnetic polarity, but that is about their only similarity. In the KS model the field is in the same direction throughout and the weight of the plasma causes the field lines to dip, hence containing the plasma. The plasma in the KR model is held between two oppositely directed fields, and the lines on top and bottom run in different directions

case reached a steady state. Malherbe and Priest studied the effects of photospheric flows on prominence sheets supported by potential magnetic fields modeled by various complex functions.

In this paper a one dimensional flow as a boundary condition will be used to generate a potential magnetic field in a current free region above the flow, a special case of a force free region $(\nabla \times \boldsymbol{B}=\alpha \boldsymbol{B})$ with $\alpha=0$. In future papers full two-dimensional convection will create magnetic configurations in a current free region above the flow. 


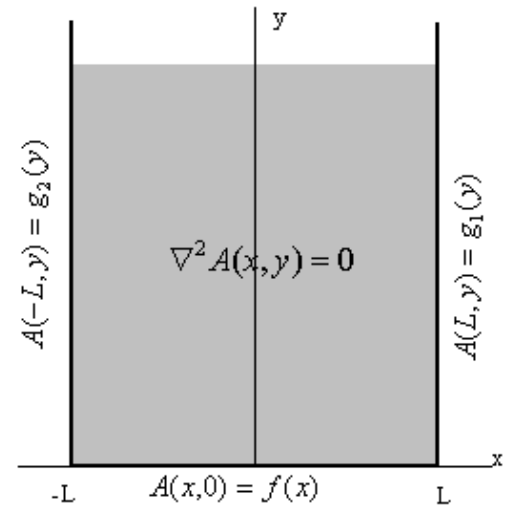

(a)

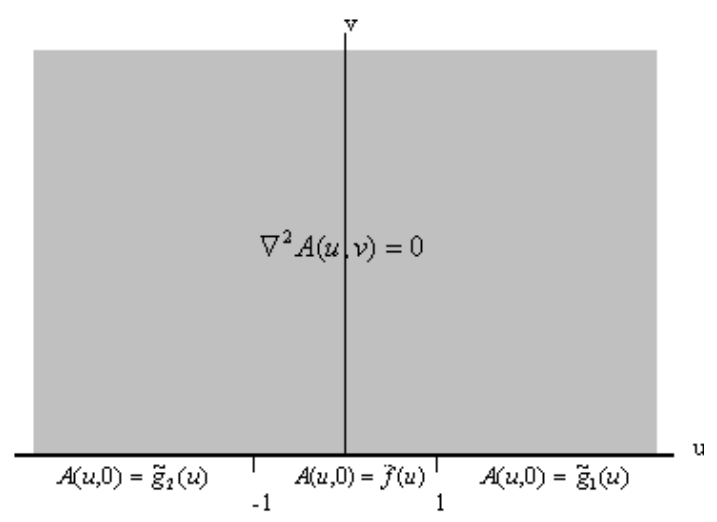

(b)

Fig. 4. The $x y$ and $u v$-planes

\section{The mathematical problem}

To build a magnetic field in a current free region above a one-dimensional flow simulating the top of a convection cell, it is necessary to solve $\boldsymbol{\nabla} \times \boldsymbol{B}=\mathbf{0}$ in a semi-infinite strip $|x| \leq L, y \geq 0$ where $y=0$ is the photosphere. Due to the two-dimensional nature of the problem we can simplify by using the vector potential $A(x, y)$ for $\boldsymbol{B}$. Then $\boldsymbol{B}=\left\langle\frac{\partial A}{\partial y},-\frac{\partial A}{\partial x}, 0\right\rangle, A$ must satisfy Laplace's equation $\nabla^{2} A=0$, and the field lines are given by $A(x, y)=$ constant. At $x=L$ and $x=-L$ let $A(x, y)$ be $g_{1}(y)$ and $g_{2}(y)$ respectively, and the field potential at the photosphere will be denoted by $f(x)$ (see Fig. 4a). This problem can be solved by mapping the strip to a half-plane plane (see Fig. 4b), then using the method of images.

\section{Solution by conformal mapping}

Laplace's equation is invariant under a conformal mapping, and the transformation $w=\sin \left(\frac{\pi z}{2 L}\right)$ where $z=x+i y$ and $w=u+i v$ maps the semi-infinite strip to the halfplane (Churchill 1984). The problem in the $u v$-plane becomes, solve $\nabla^{2} A(u, v)=0$ in the half-plane $-\infty<u<$ $\infty, v>0$ where:

$A(u, 0)=\left\{\begin{array}{rrr}\tilde{g}_{2}(u), & u<-1 \\ \tilde{f}(u) & , \quad-1<u<1 \\ \tilde{g}_{1}(u), & u>1 .\end{array}\right.$

This can be solved by the method of images (Haberman 1998) to find:

$A(u, v)=\frac{v}{\pi} \int_{-\infty}^{\infty} \frac{A(s, 0)}{(s-u)^{2}+v^{2}} \mathrm{~d} s$.

Finally, by using the inverse transformation and rewriting the integral we find:

$$
\begin{aligned}
A(x, y)= & \frac{v}{\pi}\left[\int_{-\infty}^{-1} \frac{\tilde{g}_{2}(s)}{(s-u)^{2}+v^{2}} \mathrm{~d} s\right. \\
& +\int_{-1}^{1} \frac{\tilde{f}(s)}{(s-u)^{2}+v^{2}} \mathrm{~d} s \\
& \left.+\int_{1}^{\infty} \frac{\tilde{g}_{1}(s)}{(s-u)^{2}+v^{2}} \mathrm{~d} s\right]
\end{aligned}
$$

where:

$$
\begin{aligned}
u & =\sin \left(\frac{\pi x}{2 L}\right) \cosh \left(\frac{\pi y}{2 L}\right) \\
v & =\cos \left(\frac{\pi x}{2 L}\right) \sinh \left(\frac{\pi y}{2 L}\right) \\
\tilde{f}(s) & =f\left(\frac{2 L}{\pi} \sin ^{-1} \mathrm{~s}\right) \\
\tilde{g}_{1}(s) & =g_{1}\left(\frac{2 L}{\pi} \cosh ^{-1} \mathrm{~s}\right) \\
\tilde{g}_{2}(s) & =g_{2}\left(\frac{2 L}{\pi} \cosh ^{-1}(-\mathrm{s})\right) .
\end{aligned}
$$

If we assume complete symmetry about $x=0$, i.e. $g_{1}(y)=$ $g_{2}(y)=g(y)$, which physically would imply $f(-x)=f(x)$, then the solution can be reduced to:

$$
\begin{aligned}
A(x, y)= & \frac{v}{\pi}\left[\int_{-1}^{1} \frac{\tilde{f}(s)}{(s-u)^{2}+v^{2}} \mathrm{~d} s+\int_{1}^{\infty} \frac{\tilde{g}(s)}{(s+u)^{2}+v^{2}}\right. \\
& \left.+\frac{\tilde{g}(s)}{(s-u)^{2}+v^{2}} \mathrm{~d} s\right]
\end{aligned}
$$

where:

$$
\begin{aligned}
u & =\sin \left(\frac{\pi x}{2 L}\right) \cosh \left(\frac{\pi y}{2 L}\right) \\
v & =\cos \left(\frac{\pi x}{2 L}\right) \sinh \left(\frac{\pi y}{2 L}\right) \\
\tilde{f}(s) & =f\left(\frac{2 L}{\pi} \sin ^{-1} \mathrm{~s}\right) \\
\tilde{g}(s) & =g\left(\frac{2 L}{\pi} \cosh ^{-1} \mathrm{~s}\right) .
\end{aligned}
$$

\section{Some special cases}

First we examine some cases where the integrals can be evaluated exactly. One such case is $f(x)=\alpha$ and $g(y)=\beta$ where $\alpha$ and $\beta$ are constants. The solution here is:

$$
A(x, y)=\frac{2}{\pi}(\alpha-\beta) \tan ^{-1}\left[\frac{\cos \frac{\pi}{2 L} x}{\sinh \frac{\pi}{2 L} y}\right]+\beta \text {. }
$$




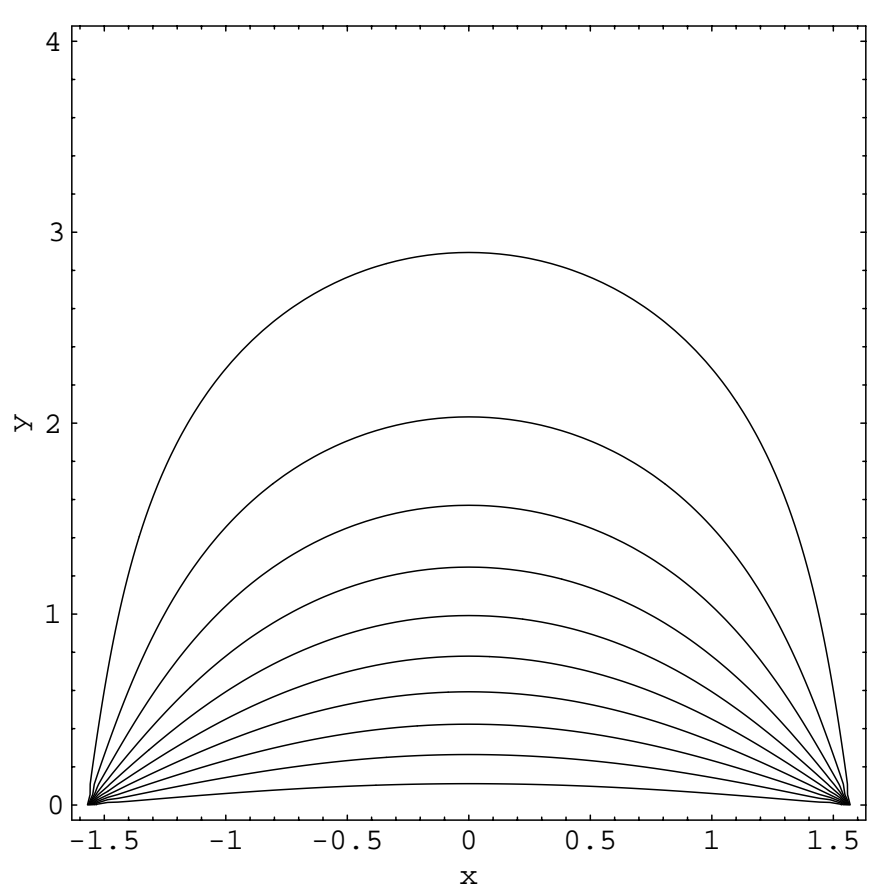

Fig. 5. $f(x)=1$ and $g(y)=0$

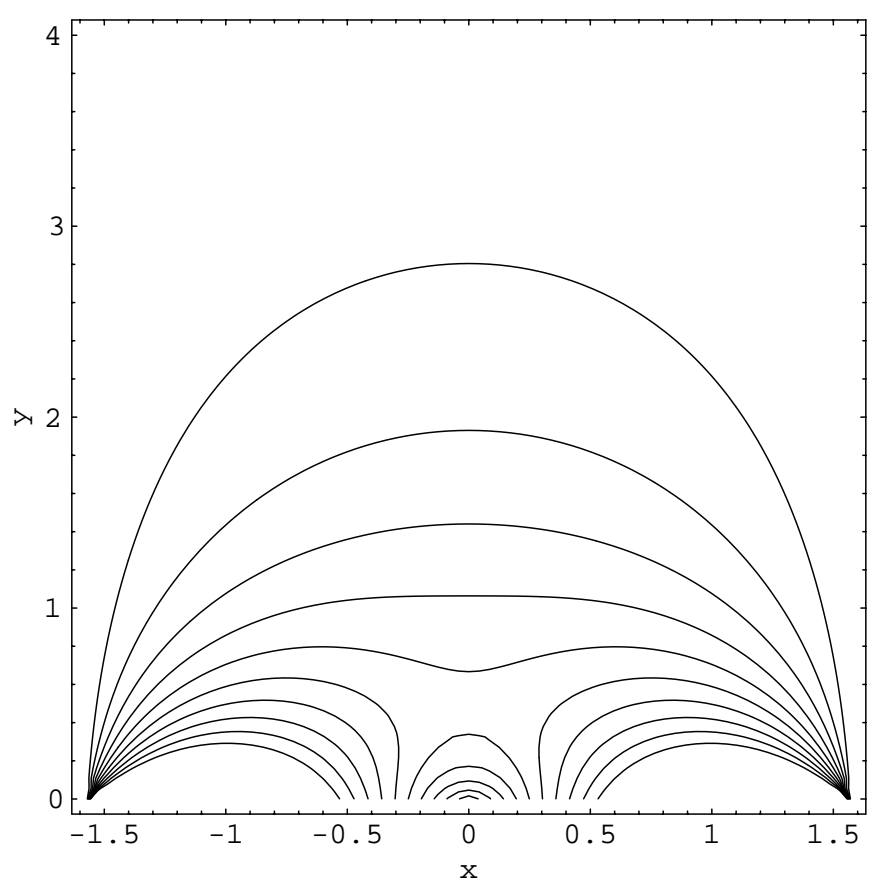

Fig. 6. $f(x)=|\sin x|$ and $g(y)=0$

The contour plot of $A(x, y)$, with $L=\frac{\pi}{2}$, gives simple magnetic arches (see Fig. 5). These arches could be a KS magnetic configuration before any plasma has condensed out of the corona to bend the field lines.

We now examine the case where $f(x)=|\sin x|$ and $g(x)=0$. The solution here can be found exactly but is extremely complicated so will not be presented. The contour plot resembles a KR topology (again with $L=\frac{\pi}{2}$ ), see Fig. 6.

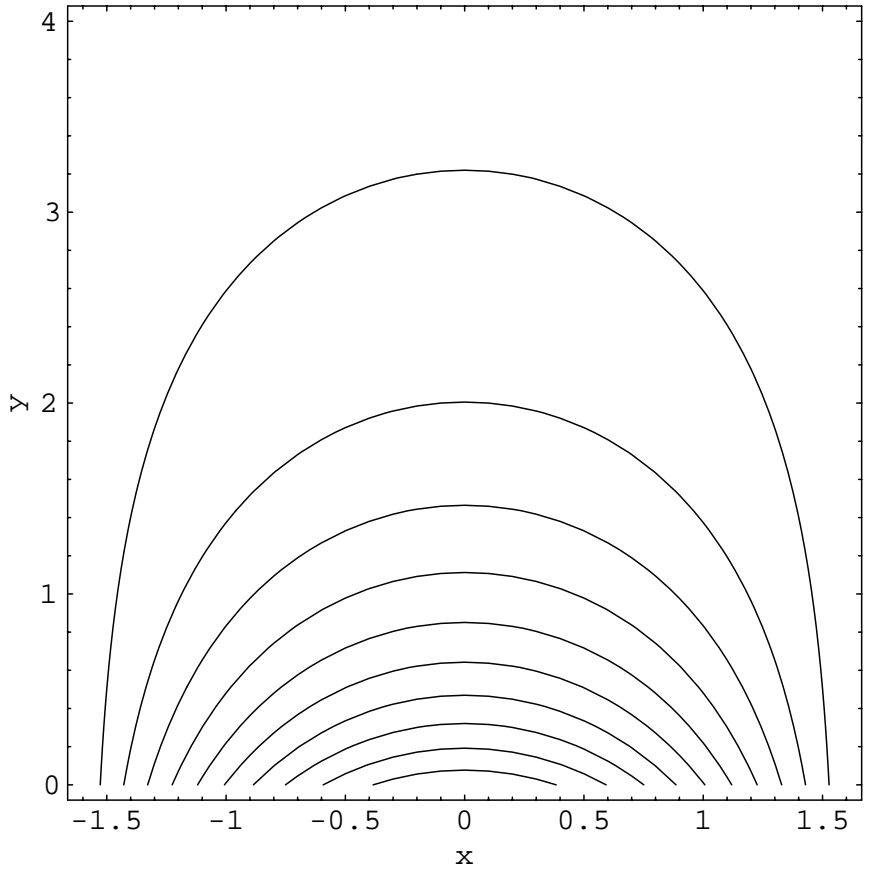

Fig. 7. $f(x)=\cos x$ and $g(y)=0$

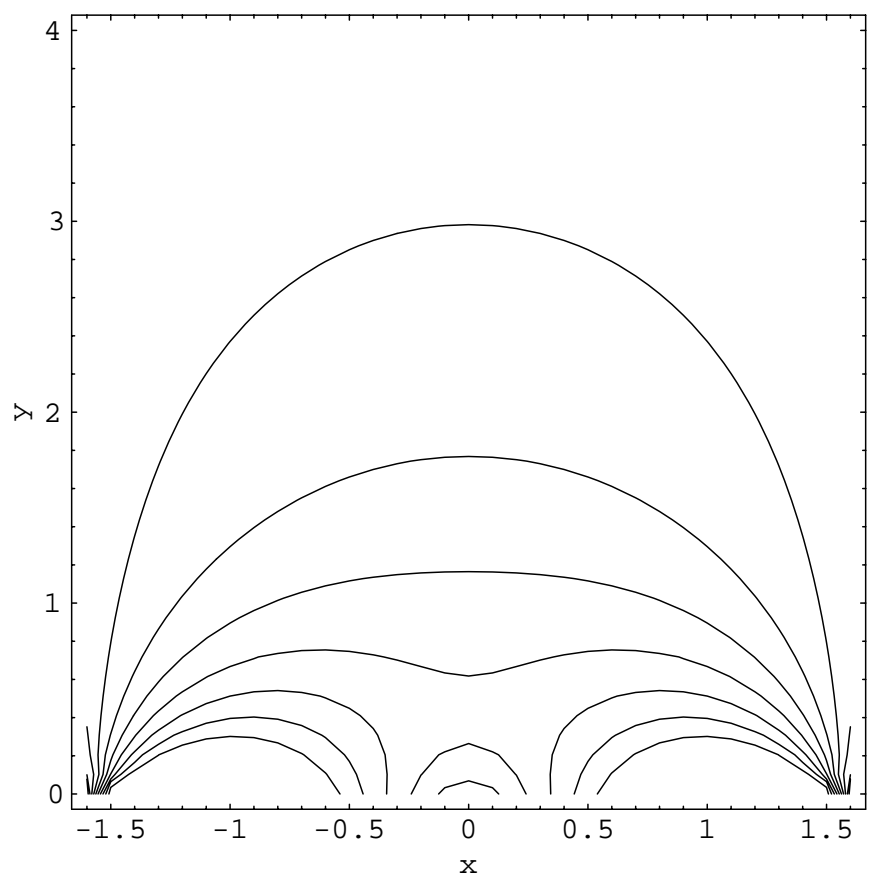

Fig. 8. $f(x)=\left|\frac{2 x}{\pi}\right|$ and $g(y)=0$

The previous case corresponds to a lower boundary potential that is weak at the center of the cell and gets stronger towards the vertices. The potential $f(x)=\cos x$ does the opposite, and gives the magnetic field lines in Fig. 7, computed numerically.

The magnetic field lines for $f(x)=\left|\frac{2}{\pi} x\right|$ and $f(x)=$ $1-\left|\frac{2}{\pi} x\right|$ are Figs. 8 and 9 respectively.

Now let's examine a case where $g(y) \neq 0$. The contour plots in Figs. 10 and 11 are $f(x)=|\sin x|$ and $f(x)=1$ respectively, both with $g(y)=\left\{\begin{array}{ll}1, & 0<y<.5 \\ 0, & y>.5\end{array}\right.$. Note how 


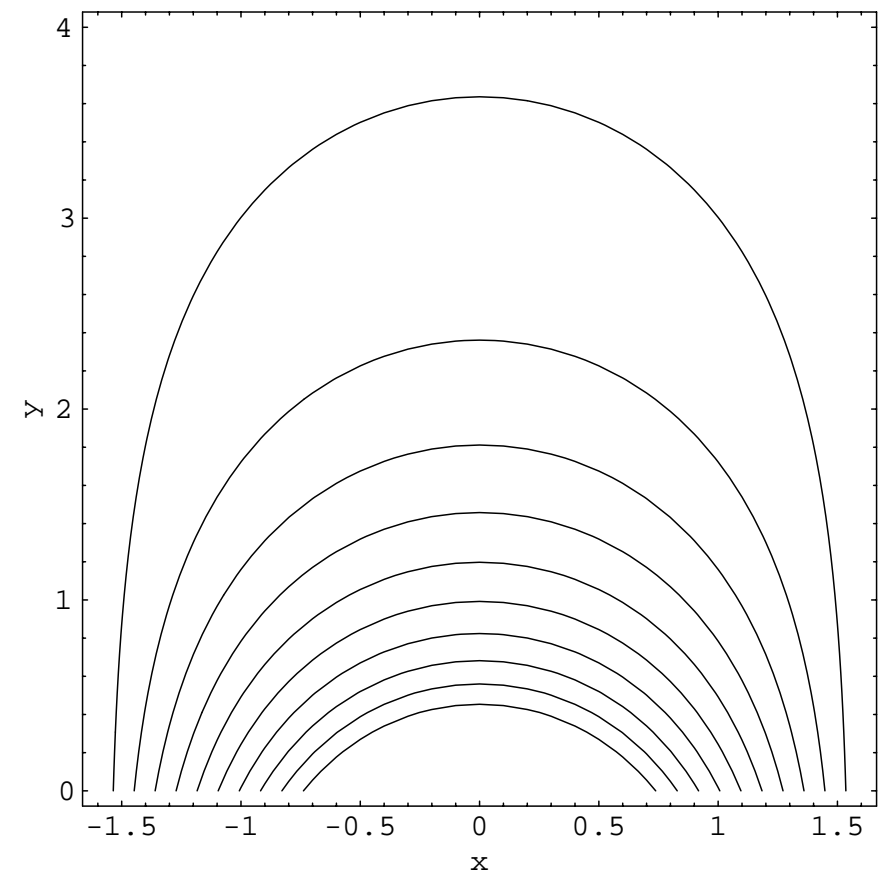

Fig. 9. $f(x)=1-\left|\frac{2 x}{\pi}\right|$ and $g(y)=0$

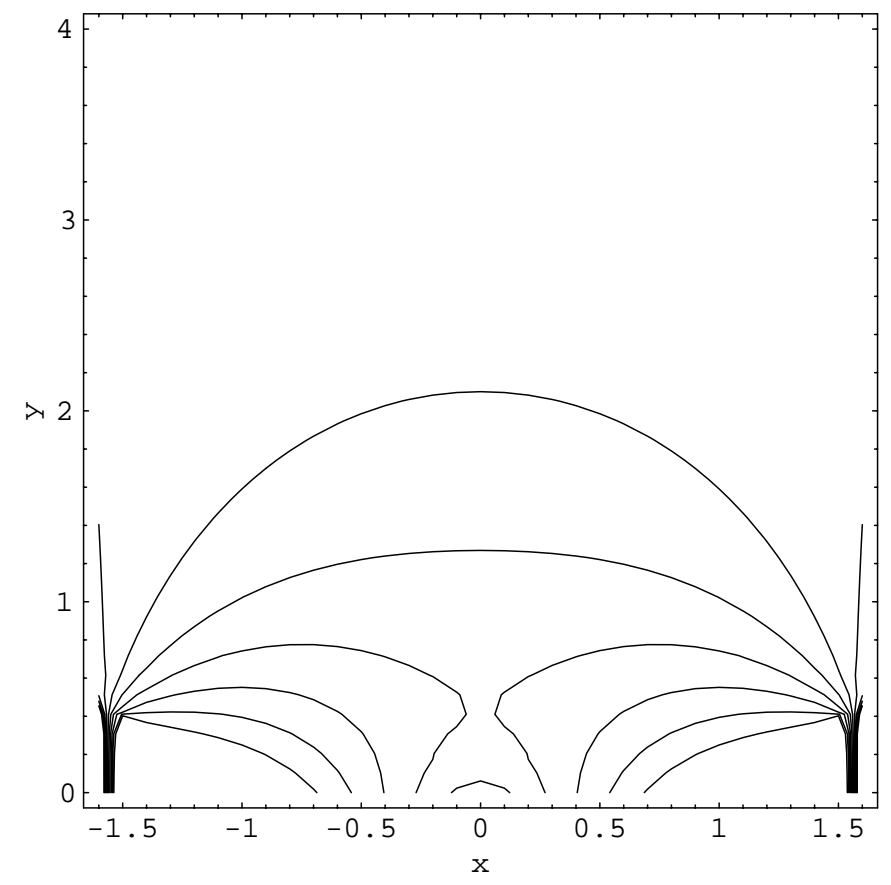

Fig. 10. $f(x)=|\sin x|$ and $g(y)=1$ if $0<y<.5,0$ if $y>.5$

the field lines bunch together near the boundary to form "feet".

\section{Conclusion}

It appears that photospheric potentials that increase away from the origin create KR type configurations and ones that decrease form KS type. Whether or not this is a

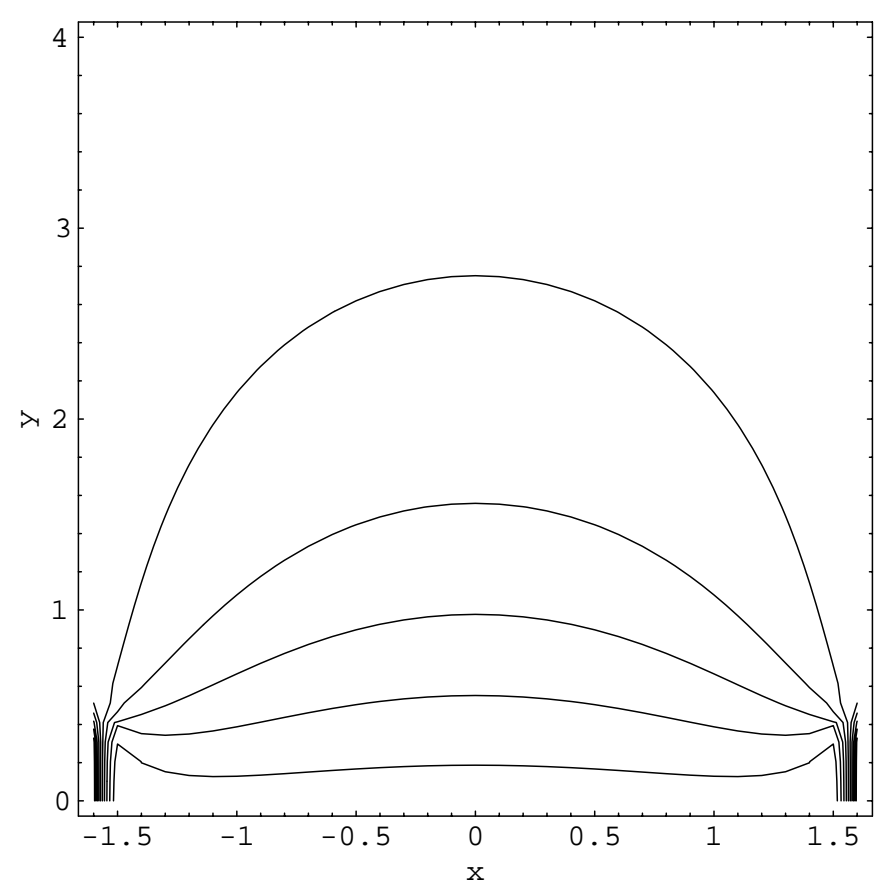

Fig. 11. $f(x)=1$ and $g(y)=1$ if $0<y<.5,0$ if $y>.5$

general connection remains to be seen. Of course the magnetic field in the corona is not just formed by a line current at the photosphere and for a more realistic model the convection has to be made fully two-dimensional. This is ongoing work and will be the subject of a future paper.

Acknowledgements. This paper was written under the auspices of the Department of Mathematics and Statistics, Old Dominion University, Norfolk VA. I am grateful to Dr. John Adam, my Ph.D. advisor and the referee for their suggestions on improving this article.

\section{References}

Ballester, J. L., \& Priest, E. R. 1987, Solar Phys., 109, 335 Churchill, R. V., \& Brown, J. W. 1984, Complex Variables with Applications (McGraw-Hill Book Co. Inc., NY)

Haberman, R. 1998, Elementary Applied Partial Differential Equations (Prentice-Hall, Inc., NJ)

Kippenhahn, R., \& Schlüter, A. 1957, Z. Astrophys., 43, 36

Kuperus, M., \& Raadu, M. A. 1974, A\&A, 31, 189

Malherbe, J. M., \& Priest, E. R. 1983, A\&A, 123, 80

Milne, A. M., Priest, E. R., \& Roberts, B. 1979, ApJ, 232, 304

Parker, E. N. 1963, ApJ, 138, 552

Poland, A., \& Anzer, U. 1971, Solar Phys., 19, 401

Priest, E. R. 1982, Solar Magnetohydrodynamics (D. Reidel Publ. Co., Dordrecht, Holland)

Priest, E. R., Hood, A. W., \& Anzer, U. 1989, ApJ, 344, 1010 Tandberg-Hanssen, E. 1974, Solar Prominences (D. Reidel Publ. Co., Dordrecht, Holland)

Tandberg-Hanssen, E. 1979, in Physics of Solar Prominences, ed. E. Jensen et al., IAU Colloq., 44, 138

Weiss, N. O. 1966, Proc. Roy. Soc. Lond. A., 293, 310 